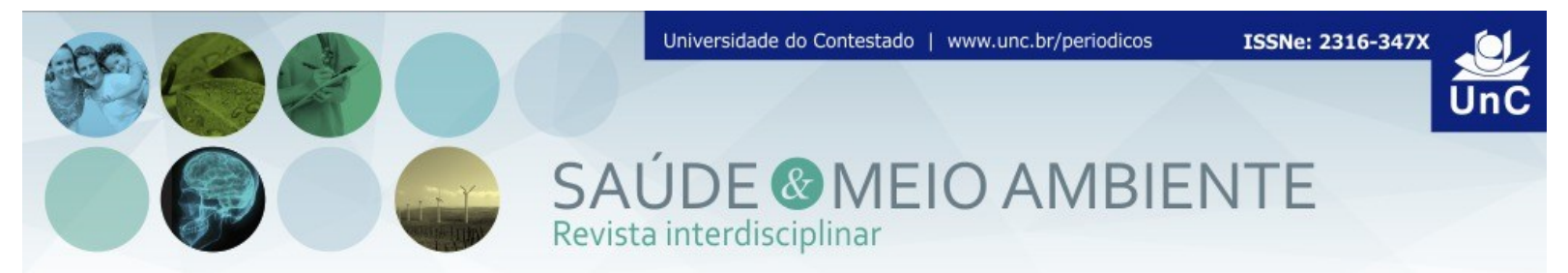

\title{
QUALIDADE MICROBIOLÓGICA DE CHÁ VERDE (CAMELLIA SINENSIS) COMERCIALIZADO NO MUNICÍPIO DE CONCÓRDIA - SC
}

Francieli Dalle Laste Valmorbida ${ }^{1}$

\begin{abstract}
RESUMO: As plantas medicinais estão a cada dia sendo mais utilizadas como preventivos de doenças e até mesmo na cura de enfermidades, visto que a flora medicinal constitui um arsenal terapêutico de enorme importância. A utilização de plantas medicinais encontra-se em expansão, no entanto, a qualidade de produtos à base de plantas é muito precária, diante disso a qualidade microbiológica de plantas medicinais deve ser levada em consideração, uma vez que contaminações por microrganismos patogênicos podem acarretar sérias consequências à saúde de quem as utiliza. O presente projeto objetivou realizar análises microbiológicas de chá comercializado em sachê em um supermercado. Coletou-se uma amostra de chá verde (Camellia sinensis) em um supermercado no município de Concórdia - SC e encaminhou-se para análise em um laboratório localizado no Oeste de Santa Catarina. De acordo com os resultados das análises o nível encontrado de contaminação para microrganismos aeróbios totais foram de $3,0 \times 10^{2} \mathrm{UFC} / \mathrm{g}$, para bolores e leveduras o resultado foi de $1,0 \times 10^{2} \mathrm{UFC} / \mathrm{g}$. Ainda a amostra apresentou um valor de $1,0 \times 10^{1} \mathrm{UFC} / \mathrm{g}$ para coliformes totais, $1,0 \times 10^{2} \mathrm{UFC} / \mathrm{g}$ para Staphylococcus coagulase positiva e para Salmonella sp. o resultado foi negativo. Portanto, baseado nos resultados obtidos para contaminação por aeróbios totais, bolores, leveduras e Salmonella sp., a amostra analisada apresenta-se em boas condições para consumo. Para os demais contaminantes a legislação não estabelece os valores máximos permitidos.
\end{abstract}

Palavras-chave: Plantas medicinais. Camellia sinensis. Microbiologia

\section{MICROCIOLOGICAL QUALITY GREEN TEA (Camellia sinensis) COMMERCIALISED IN THE CITY OF CONCÓRDIA-SC}

\begin{abstract}
Medicinal plants are every day being more used as preventive diseases and even cure of diseases, since the medicinal flora is a therapeutic arsenal of enormous importance. The use of medicinal plants is growing, however, the quality of herbal products is very precarious, given that the microbiological quality of medicinal plants should be taken into consideration, since contamination by pathogenic microorganisms can cause serious consequences to the health of those who use them. This project aimed to perform microbiological analysis of tea sold in sachet in a supermarket. Gathered up a green tea sample (Camellia sinensis) in a supermarket
\end{abstract}

${ }^{1}$ Graduada em Ciências Biológicas (Bacharel e Licenciatura) pela Universidade do Contestado -
Campus Concórdia. Itá, Santa Catarina. Brasil. E-mail: francieli dallelaste@hotmail.com 

$-\mathrm{SC}$

in the city of Concórdia - SC and sent for analysis in a laboratory located in the West of Santa Catarina. According to the test results the level of contamination found for total aerobic microorganisms were $3.0 \times 10^{2} \mathrm{UFC} / \mathrm{g}$, molds and yeasts for the result was $1.0 \times 10^{2} \mathrm{UFC} / \mathrm{g}$. Although the sample showed a value of $1.0 \times 10^{1} \mathrm{UFC} / \mathrm{g}$ for total coliforms, $1.0 \times 10^{2} \mathrm{UFC} / \mathrm{g}$ for coagulase positive Staphylococcus and Salmonella sp. the result was negative. Therefore, based on the results obtained for total aerobic contamination, molds, yeasts and Salmonella sp., The sample analyzed is presented in good condition for consumption. For other contaminants legislation does not establish the maximum allowable values.

Keywords: Medicinal plants. Camellia sinensis. Microbiology.

\title{
INTRODUÇÃO
}

Desde os primórdios, os homens buscam na natureza recursos para melhorar suas condições de vida, sendo que a utilização de plantas medicinais sempre existiu como alimento, aumentando desta forma suas chances de sobrevivência (LORENZI; MATOS, 2002).

O Brasil era um país essencialmente rural, com amplo uso da flora medicinal até o século XX. Porém, com o início da industrialização, esse conhecimento foi deixado de lado, sendo considerado sinônimo de atraso tecnológico e muitas vezes charlatismo, enfatizando o acesso a medicamentos industrializados (LORENZI; MATOS, 2002).

Depois de quase meio século de predomínio da medicina alopática e dos remédios sintéticos fabricados em laboratório, a Ciência volta a atenção para medicamentos naturais, pelo fato de as pessoas estarem substituindo drogas compradas em farmácia por remédios caseiros devido à preocupação com os efeitos colaterais provocados pela utilização dos mesmos (RIGON; MENUTE, 1996).

Segundo Lorenzi e Matos (2002, p.17):

\begin{abstract}
Um segundo aspecto que certamente contribuiu para o afastamento do estudo das plantas medicinais e o restante da ciência foi a ampla resistência desta primeira às profundas alterações que tanto a sistemática vegetal quanto a medicina experimentaram no final do século XIX e todo o século $\mathrm{XX}$. Fortemente baseado em trabalhos mais clássicos, o estudo das plantas medicinais mostrou uma resistência inicial a acompanhar as grandes revoluções científicas ocorridas neste período. Essa inadequação inicial manteve a fitoterapia em um período de obscurantismo, onde esteve mais próximo do misticismo que da ciência.
\end{abstract}

Em 1978 a Assembleia Geral da Organização Mundial de Saúde deu início a um programa no qual se dava ênfase a utilização de plantas medicinais. $O$ estudo das plantas medicinais começou a ser incentivado e no Brasil a Central de Medicamentos (CEME) elaborou uma lista das plantas que deveriam ser 
pesquisadas com fins medicinais. Porém, a grande variedade de espécies vegetais no Brasil, voltou a atenção de pesquisadores do mundo inteiro (OLIVEIRA; AKISUE, 2000).

As ervas e outros remédios naturais dão resultado, segundo muitos pacientes, médicos e pesquisadores científicos. Desde que sejam seguros e usados de forma adequada, são tão eficazes quanto os medicamentos convencionais (CARPER, 1998).

As plantas medicinais estão intimamente ligadas à botânica, uma vez que muitos dos primeiros trabalhos que buscavam nomear e categorizar os vegetais tinham como objetivo principal oferecer um catálogo conciso de plantas com importância medicinal. Uma dessas importantes obras foi De Matéria Medica, que catalogou e ilustrou cerca de 600 diferentes plantas usadas para fins medicinais, sendo que muitos dos nomes que foram apresentados ainda hoje são usados na botânica (LORENZI; MATOS, 2002).

É de suma relevância destacar que a flora medicinal constitui um arsenal terapêutico de enorme importância. Há vários séculos têm sido consideradas fontes medicamentosas empregadas tanto em preparações tradicionais quanto, mais recentemente, na forma de princípios ativos puros, porém, algumas plantas são conhecidas por serem bastante tóxicas ao organismo (CORREA;; BATISTA; QUINTAS, 1998).

Há um grande interesse científico no desenvolvimento da pesquisa das plantas que são utilizadas na medicina popular, isso porque, a indústria farmacêutica possui elevados custos para fabricar remédios além de possuir dificuldade em se obter assistência médica e farmacêutica de qualidade e a própria confiança e aceitação da população nos produtos naturais, estão superexpandindo o processo de utilização de plantas medicinais tanto na terapêutica quanto na cosmetologia. Porém, são necessários estudos criteriosos a fim de que haja comprovação ou não de seus efeitos terapêutico-farmacológicos e seus possíveis efeitos colaterais (CORRÊA; BATISTA; QUINTAS, 1998).

Atualmente pode-se encontrar inúmeras espécies de plantas medicinais. Essas plantas estão à disposição da população em forma de xaropes, tinturas, os quais são feitos a partir da retirada do extrato das plantas ou também chamado de princípio ativo (MAURY; RUDDER, 2002).

As plantas medicinais estão também disponíveis na sua forma natural, para que se possa preparar em casa os diversos tipos de chás (MAURY; RUDDER, 2002).

As plantas medicinais agem bem progressivamente, são de pouca utilidade em caso de crise aguda. São indicadas para tratamentos preventivos, de manutenção e podem ser muito importantes auxiliares da ação de um medicamento forte (MAURY; RUDDER, 2002). 
Segundo Sossae (2013) após a obtenção das plantas medicinais, normalmente pode ser feito: uso direto da planta fresca, extração de substâncias ativas ou aromáticas da planta fresca bem como secagem da planta.

As regulamentações de cultivo, manejo, produção, distribuição e uso de plantas medicinais visam assegurar a qualidade e eficácia do produto final. No entanto, para garantir essa qualidade, deve-se abranger e garantir o tratamento adequado em todas as fases de toda cadeia produtiva. Devem ser observados os diferentes sistemas de produção e técnicas nas diferentes regiões. Além dos aspectos botânicos, químicos e farmacológicos (BRASIL. MINISTÉRIO DA SAÚDE, 2007).

Uma das maiores preocupações do ser humano visa a manutenção da saúde. Para isso, muitas são as atitudes a serem tomadas a fim de ter uma vida saudável. Ter uma alimentação equilibrada, praticar exercícios físicos regularmente, combater o estresse, diminuir a exposição a riscos ambientais - tais como ruídos, poluição, radiação solar, contribuem muito para manter a saúde (CORRÊA; BATISTA; QUINTAS, 1998).

Segundo Oliveira e Akisue (2000, p.157): "Planta medicinal é todo vegetal que contém em um ou vários de seus órgãos substâncias que podem ser empregadas para fins terapêuticos ou precursores de substâncias utilizadas para tais fins."

As plantas medicinais contribuíram muito desde a antiguidade, quando as pessoas utilizavam as plantas para o tratamento de doenças humanas e dos animais. Porém, houve uma época que a utilização de plantas medicinais e fitoterápicos parecia estar decaindo devido a indústria farmacêutica produzir os mais diversos medicamentos eficazes na cura de várias enfermidades (OLIVEIRA; AKISUE, 2000).

A utilização de plantas medicinais encontra-se em expansão, no entanto, a qualidade de produtos à base de plantas é muito precária (SEBRAE, 2010). Por este motivo, a qualidade microbiológica de plantas medicinais deve ser levada em consideração, uma vez que contaminações por microrganismos patogênicos podem acarretar sérias consequências à saúde de quem as utiliza.

É importante destacar que o corpo humano é continuamente habitado por diversos microrganismos, os quais são inofensivos, podendo até mesmo ser benéficos (HARVEY; CHAMPE; FISCHER, 2008). No entanto, é necessário estar atento as possíveis infecções causadas por microrganismos patogênicos, os quais podem acarretar sérias consequências a saúde.

Levando em consideração que o chá verde (Camellia sinensis) tem sido muito utilizado como alimento anti-inflamatório e antioxidante, pois previne 0 envelhecimento e doenças como o câncer, podem diminuir ainda o colesterol ruim (RBS, 2013), o presente trabalho objetivou realizar análises microbiológicas de chá verde (Camellia sinensis) comercializado em sachês em um supermercado de Concórdia-SC, investigando a presença de bolores e leveduras, coliformes totais, Staphylococcus coagulase positiva, mesófilos aeróbios estritos facultativos e Samonella sp. 


\section{MATERIAL E MÉTODOS}

No Brasil, apesar da grande utilização de produtos à base de plantas medicinais, desde a antiguidade, não era realizado nenhum tipo de controle. Até que em 1995, o Ministério da Saúde instituiu em sua portaria $n^{\circ}$ 6/MS/SNVS, de 31 de janeiro de 1995 o registro dos produtos fitoterápicos junto ao sistema de vigilância sanitária para sua comercialização (BRASIL, 1995).

Após, surgiu a RDC 17 (BRASIL, 2000), A RDC 48 (BRASIL, 2004) que regulamentam o registro dos fitoterápicos, assegurando eficácia e qualidade em todos os produtos. É importante destacar que o registro dos produtos fabricados através de plantas medicinais são relevantes, pois se as plantas estiverem contaminadas, os fitoterápicos serão automaticamente influenciados.

As plantas medicinais podem ser comercializadas de acordo com a Lei $\mathrm{n}^{\circ}$ $5.991 / 73$, enquanto que os produtos comercializados a partir das mesmas devem ser registrados junto a ANVISA (CARVALHO et al., 2007).

Em todos os ecossistemas os microrganismos podem ser encontrados, inclusive em associações com os organismos multicelulares. Aqueles que habitam o corpo humano fazem parte da flora normal e são considerados passageiros benignos. Podem também ter importante função, como por exemplo as bactérias que atuam na degradação do conteúdo intestinal. Porém, é importante destacar que bactérias, fungos, protozoários, helmintos e vírus muitas vezes são prejudiciais aos seres humanos, produzindo compostos tóxicos ou causando alguma infecção de forma direta (STROHL; ROUSE; FISCHER, 2008).

Considerando a preocupação com a qualidade das plantas medicinais comercializadas, o presente projeto objetivou a análise microbiológica de uma amostra de Camellia sinensis coletada em um supermercado no município de Concórdia-SC no mês de dezembro do ano de dois mil e treze.

As análises foram realizadas em um laboratório da região oeste de Santa Catarina devidamente credenciado pela CIDASC, sendo que o laboratório utilizou a seguinte metodologia para análises microbiológicas: Brasil. Ministério da Agricultura, Pecuária e Abastecimento. Secretaria da Defesa Agropecuária. Métodos analíticos oficiais para controle de produtos de origem animal e água. Instrução Normativa $n^{\circ}$ 62, de 26 de agosto de 2003 e AOAC OMA 2013. 


\section{RESULTADOS E DISCUSSÕES}

A avaliação dos resultados das análises foram baseadas em World Health Organization (1998), pois as plantas medicinais possuem especificações variadas para contaminantes microbiológicos, os quais serviram de parâmetro para o resultado final.

O nível encontrado de contaminação por microrganismos aeróbios foi de $3,0 \mathrm{x}$ $10^{2} \mathrm{UFC} / \mathrm{g}$, sendo que a especificação da OMS é de no máximo $5,0 \times 10^{7} \mathrm{UFC} / \mathrm{g}$ para materiais vegetais destinados ao uso na forma de chás e infusões e de no máximo $5,0 \times 10^{5} \mathrm{UFC} / \mathrm{g}$ para materiais vegetais destinados ao uso interno (WHO, 1998).

A contaminação por bolores e leveduras da amostra analisada foi de $1,0 \times 10^{2}$ UFC/g, sendo que a especificação da OMS é de no máximo 5,0 x10 4 UFC/g para materiais vegetais destinados ao uso na forma de chás e infusões e no máximo $5,0 \mathrm{x}$ $10^{3} \mathrm{UFC} / \mathrm{g}$ para vegetais destinados ao uso interno (WHO, 1998).

Ainda, a amostra analisada apresentou um valor de $1 \times 10^{1} \mathrm{UFC} / \mathrm{g}$ de coliformes totais, no entanto a legislação não estabelece limite máximo para coliformes totais.

A contaminação para Staphylococcus coagulase positiva foi de $1 \times 10^{2} \mathrm{UFC} / \mathrm{g}$. De acordo com Abba, et al. (2009); Franco e Landgraf (2008); Rocha et al. (2010); Wannipa et al. (2007) apud Rocha et al. (2014):

\footnotetext{
Apesar da inexistência de limites estabelecidos pela OMS (Organização Mundial de Saúde) para a presença em plantas medicinais do $S$. aureus ou outros estafilococus coagulase positivos, a detecção de populações significativas da espécie, grupo neste material não deve ser desprezada, existindo relatos de sua detecção e do risco que representam aos consumidores.
}

Em relação a contaminação por Salmonella sp. o resultado foi negativo, não sendo detectada esta bactéria na amostra analisada, estando em acordo com a Organização Mundial de Saúde. É importante ressaltar que este microrganismo é frequentemente envolvido em casos e surtos de doenças de origem alimentar em diversos países, inclusive no Brasil (FRANCO; LANDGRAF, 1996).

As plantas medicinais geralmente possuem contaminações por bactérias e fungos provenientes do solo (WHO, 1998). É importante ter maior atenção na coleta e armazenamento e secagem das plantas a fim de obter-se um produto de melhor qualidade para o consumidor final (SOSSAE, 2009).

De acordo com as análises microbiológicas realizadas para microrganismos aeróbios totais e bolores e leveduras e Salmonella sp., a amostra analisada apresenta-se em boas condições para consumo, tanto para uso na forma de chás e infusões quanto para o uso interno. 
No entanto, a legislação não determina valores máximos para Staphylococcus coagulase positiva e coliformes totais, ficando desta forma duvidosa a qualidade final desta amostra de chá.

É importante ressaltar que práticas inadequadas no momento da coleta, manipulação e produção final podem causar contaminações e posteriormente crescimento microbiano (WORLD HEALTH ORGANIIZATION, 1998). Por este motivo, acredita-se que haja a necessidade de treinamentos as pessoas que fazem parte de todo ciclo de produção dos chás para garantir qualidade satisfatória ao consumidor final, pois contaminações por bactérias e fungos podem acarretar sérios problemas na saúde de quem utiliza essas plantas.

\section{REFERÊNCIAS}

BRASIL. MINISTÉRIO DA SAÚDE. Programa nacional de plantas medicinais e fitoterápicos. 2007. Disponível em:

$<$ http://portal.mda.gov.br/portal/saf/arquivos/view/Programa_Nacional_de_Plantas_M edicinais_e_Fitoter\%C3\%A1picos..pdf> Acesso em: 06. jan 2014

Portaria $n^{\circ}$ 6/MS/SNVS, de 31 de janeiro de 1995. Disponível em: < http://www.anvisa.gov.br/... > Acesso em: 20. jan 2014

. Resolução - RDC n 17, de 24 de fevereiro de 2000. Disponível em: < http://www.anvisa.gov.br/legis/resol/2000/17_00rdc.htm> Acesso em: 20. jan 2014

. Resolução - RDC $\mathbf{n}^{\circ}$ 48, de 16 de março de 2004. Disponível em:

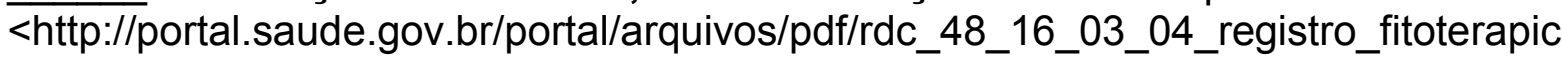
os\%20.pdf> Acesso em: 10. jan 2014

CARPER, Jean. Curas milagrosas: descobertas científicas que revelam o poder de cura das ervas, vitaminas e de outros remédios naturais. 2. ed. Rio de Janeiro:

Campus, 1998.

CORREAA, Anderson Domingues; BATISTA, Rodrigo Siqueira; QUINTAS, Luis Eduardo M. Plantas medicinais: do cultivo à terapêutica. 5. ed. Rio de Janeiro: Vozes, 1998.

CARVALHO et al., Aspectos da legislação no controle dos medicamentos fitoterápicos. 2007. Disponível em:

<https://portal.fucapi.br/tec/imagens/revistas/004_rev011_aspectos_da_legislacao_n o_controle.pdf> Acesso em: 20. dez 2013.

FRANCO, B. D. G. M; LANDGRAF, M. Microbiologia dos alimentos. São Paulo: Atheneu, 1996. 
HARVEY, R A; CHAMPE, P C; FISCHER, B D. Microbiologia llustrada. 2.ed. Porto Alegre: Artmed, 2008.

LORENZI, Harri; MATOS, Francisco José de Abreu. Plantas medicinais no Brasil: nativas e exóticas cultivadas. São Paulo: Instituto Plantarum, 2002.

MAURY, E. A; RUDDER, Chantal de. Guia das plantas medicinais. São Paulo: Rideel, 2002.

OLIVEIRA, Fernando de; AKISUE, Gokithi. Fundamentos de Farmacobotânica. 2.ed. São Paulo: Atheneu, 2000.

RIGON, Giovana; MENUTE, Terezinha Lando. Plantas medicinais. 1996. Relatório de estágio (Curso de Ciências habilitação em Biologia) - Universidade do Contestado de Concórdia, Concórdia, 1996.

RBS. Cinco motivos para consumir o chá verde, mas atenção aos cuidados. 2013. Disponível em: <http://wp.clicrbs.com.br/...>. Acesso em: 06. Jan. 2014.

ROCHA, F. A. G, et al.,. Detecção de Staphylococcus aureus em plantas medicinais. IX Congresso de Iniciação científica do IFRN. Disponível em: <http:www2.ufrn.edu.br/...>. Acesso em: 17 jan. 2014.

SEBRAE. Produção de chás e extratos de plantas medicinais. Disponível em: $<$ http://www.sebrae-sc.com.br/credito/default.asp?vcdtexto=4980\&\%5E $\% 5 \mathrm{E}=>$ Acesso em: 12 nov. 2010.

SOSSAE, Flávia Cristina. Plantas medicinais. Disponível em:

<http://educar.sc.usp.br/biologia/prociencias/medicinais.html>. Acesso em: 13. dez 2013.

STROHL, W; ROUSE, H; FISCHER, B. Microbiologia ilustrada. Porto Alegre: Artmed, 2008.

WORLD HEALTH ORGANIZATION. Quality control methods for medicinal plant materials. Geneva: WHO, 1998.

Artigo recebido em: 19/10/2014

Artigo aprovado em: 20/03/2016 